\title{
ON THERMAL EFFECTS IN REACTIVE WETTING
}

\author{
Andreas Mortensen ${ }^{1} *$, Fiqiri Hodaj ${ }^{2}$, and Nicolas Eustathopoulos ${ }^{2}$ \\ ${ }^{1}$ Departement of Materials, Massachusetts Institute of Technology, Cambridge, Massachusetts, USA \\ ${ }^{2}$ LTPCM-INP Grenoble, UJF, URA 29, BP 75, 38402, Saint Martin d'Hères, Cedex, France
}

(Received December 29, 1997)

(Accepted January 14, 1998)

\section{Introduction}

High-temperature capillary phenomena intervene in many material processing operations, including bonding operations such as soldering and brazing, and infiltration processes used to produce metal or ceramic matrix composites. Of particular importance in this class of processes is the issue of wetting of solids by molten inorganic materials: good wetting, as manifest by a low contact angle of the liquid on the solid in the processing environment, drives flow of the liquid over the solid and eases processing significantly. This issue has therefore motivated a large body of research aimed at developing methods for improving wetting in high-temperature systems of practical interest. Results from contact angle measurements, and also from process development work, indicate that chemical interaction between the solid and the liquid tends to promote good wetting (1-5).

Wetting is usually characterized by measurement of contact angles in sessile drop experiments, wherein a drop of the liquid is placed at fixed temperature on a smooth and flat substrate of the solid. When interfacial reactions drive wetting, these cause transitions with time in the contact angle while the triple line advances at a rate determined by the rate of reaction product formation along the substrate surface (e.g., (6-9)): reactive wetting is thus generally a dynamic phenomenon. It is customarily assumed in the interpretation of sessile drop data that the wetting process is isothermal, even in dynamic situations typical of reactive wetting; however, interfacial reactions encountered in high-temperature materials processing can be highly exothermic. These could, therefore, cause a significant increase in the local temperature at the triple line and, hence, influence both reaction kinetics and capillary forces in reactive wetting.

We examine here this influence using a simplified description of the problem, in which we take the rate of heat evolution behind the triple line of solid/liquid/vapor contact to be constant and limited in time, and the wetting process to be fully steady-state. More specifically, we address two very basic situations, encountered both in materials processes and in experimental measurements of wetting. The first is that of a liquid spreading over a bulk solid substrate, typical in particular of sessile drop experiments; the second is that of a liquid infiltrating a porous solid such as a preform of fibers or particles, or a thin capillary. We seek, in the most simple and general terms possible, to assess whether heat evolution at reacting interfaces could cause significant departures in the local temperature at the triple line.

\footnotetext{
* Departement of Materials, Swiss Federal Institute of Technology (EPFL), CH-1015, Lausanne, Switzerland.
} 


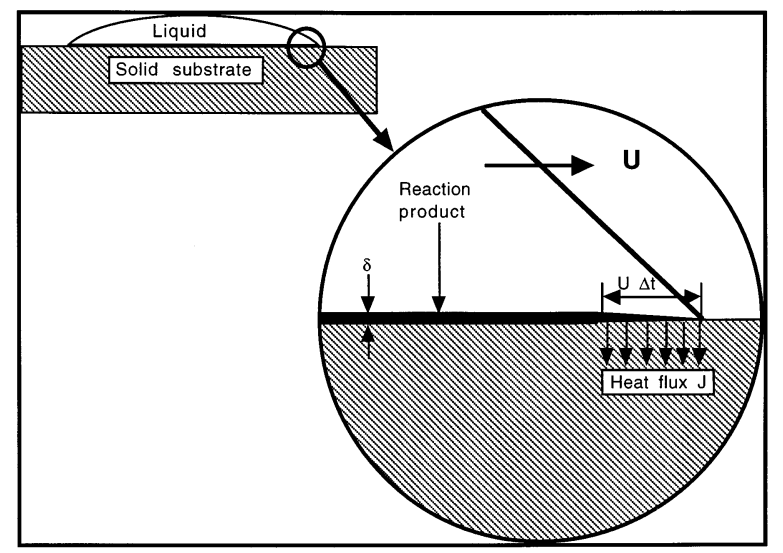

Figure 1. Assumed configuration for reaction layer formation behind the triple line in spreading over a flat substrate.

\section{Simplified Analysis of Heat Transport Near a Triple Line}

\section{Spreading Over a Bulk Substrate}

We consider a liquid/solid/gas contact line moving on a planar solid at a constant velocity $U$ equal to the rate of reaction product formation along the substrate surface (9). The chemical reaction between the liquid and the solid along the solid/liquid contact surface releases or absorbs heat while forming the reaction product. We assume that the rate of formation of the reaction product normally to the interface $(\mathrm{V})$ is constant during time $\Delta \mathrm{t}$ and negligible afterwards, such that a reaction product layer of thickness $\delta$ is formed behind the triple line over a distance $\mathrm{L}=\mathrm{U} \Delta \mathrm{t}$ along the liquid/solid interface behind the triple line, Fig. 1. This simplified picture of the problem is justified by the fact that, as a general rule, after a certain thickness of reaction product has formed the overall reaction rate becomes controlled by the slow diffusion of reactive species through the solid reaction product layer. Although $\delta$ and $\Delta \mathrm{t}$ depend on temperature, we take these parameters as given in what follows; thus, we do not address the problem of coupling between reaction rate and local temperature at the triple line.

We assume that both the solid substrate and the liquid drop are large in comparison with the zone of substantial interfacial reaction (i.e., in comparison with both $\delta$ and $\mathrm{L}$ ) and also in comparison with the zone of (potentially) raised local temperature. Therefore, far from the triple line, the temperature approaches $\mathrm{T}_{\mathrm{o}}$, the ambient experiment temperature. Also, we assume that the radius of curvature of the triple line is large in comparison with $\mathrm{L}$; we then can take the triple line to be essentially straight, thus rendering the problem two-dimensional. Finally, we also assume for simplicity that the spreading process is steady within the referential of the triple line; $U$ is thus constant, and the temperature distribution around the triple line is independent of time.

Even with these simplifications, a description of the problem is mathematically very complex in the liquid. There is a free boundary at the solid/liquid interface, the shape of which requires knowledge of local temperature and fluid velocity to be described. The convective flow pattern in the liquid is not amenable to simple analysis (e.g., (10)), and is potentially influenced by the temperature gradients generated by the reaction via Marangoni effects at the liquid surface. To approach the problem on a simple basis, we therefore temporarily make the assumption that the liquid phase absorbs no heat. This assumption is justified by the conclusion reached below, namely that under usual experimental conditions the solid substrate alone can absorb sufficient heat to prevent any rise of temperature around the triple line; a fortiori, therefore, heat transfer through the liquid then reinforces this conclusion. 


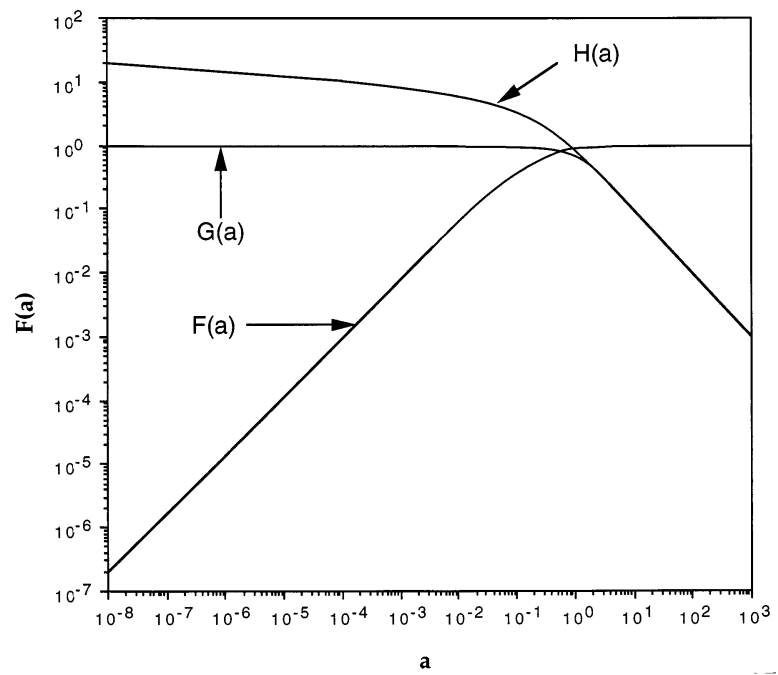

Figure 2. Plots of functions of (a) appearing in Eqs. (3), (4) and (13) respectively.

We use a referential moving with the contact line, having its $\mathrm{x}$ direction parallel to the direction of triple line motion, its $\mathrm{y}$ axis parallel to the triple line, and its $\mathrm{z}$ axis perpendicular to the solid/liquid interface, oriented into the solid. With the assumptions above, heat evolution near the triple line due to chemical interaction is assimilated to a moving strip of a constant intensity heat source, located from $\mathrm{x}=0$ to $\mathrm{x}=\mathrm{U} \cdot \Delta \mathrm{t}$, releasing $\mathrm{J}$ joules per square meter per second, and moving over a semi-infinite solid slab which is at temperature $\mathrm{T}_{\mathrm{o}}$ far from the origin in all directions. $\mathrm{J}$ is given by:

$$
\mathrm{J}=\Delta \mathrm{H} \frac{\delta}{\Delta \mathrm{t}}\left(\mathrm{J} \mathrm{m}^{-2} \mathrm{~s}^{-1}\right)
$$

where $\Delta \mathrm{H}$ is the volumetric change of enthalpy accompanying the reaction (Fig. 1).

The resulting temperature distribution in the solid is given by (11) as:

$$
\mathrm{T}=\mathrm{T}_{0}+\frac{\mathrm{J}}{\pi \lambda} \int_{0}^{\mathrm{U} \Delta \mathrm{t}} \exp \left(\frac{\mathrm{U}\left(\mathrm{x}-\mathrm{x}^{\prime}\right)}{2 \alpha}\right) \mathrm{K}_{0}\left(\frac{\mathrm{U} \sqrt{\left(\mathrm{x}-\mathrm{x}^{\prime}\right)^{2}+\mathrm{z}^{2}}}{2 \alpha}\right)
$$

where $\mathrm{K}_{\mathrm{o}}$ is the modified Bessel function of the second kind of order zero, $\lambda$ is the thermal conductivity of the substrate and $\alpha$ its thermal diffusivity. Using Eq. (11) of Ref. (11) for $\mathrm{x}=\mathrm{z}=0$, the temperature at the triple line is given by:

$$
\mathrm{T}=\mathrm{T}_{0}+\frac{2 \Delta \mathrm{H}}{\pi \rho \mathrm{c}_{\mathrm{p}}} \frac{\delta}{\mathrm{U} \Delta \mathrm{t}}\left\{\mathrm{ae}^{-\mathrm{a}}\left[\mathrm{K}_{0}(\mathrm{a})-\mathrm{K}_{1}(\mathrm{a})\right]+1\right\} \quad \text { where } \quad \mathrm{a}=\frac{\mathrm{U}^{2} \Delta \mathrm{t}}{2 \alpha}
$$

$\mathrm{K}_{1}$ is the modified Bessel function of the second kind of order one, and $\rho \mathrm{c}_{\mathrm{p}}$ is the volumetric heat capacity of the solid substrate $\left(\lambda=\rho c_{\mathrm{p}} \alpha\right)$. The term between brackets, $\mathrm{F}(\mathrm{a})=\mathrm{a} \mathrm{e}^{-\mathrm{a}}\left[\mathrm{K}_{0}(\mathrm{a})-\mathrm{K}_{1}(\mathrm{a})\right]+$ 1 , is a monotonously increasing function of a, Fig. 2. The first fraction on the right-hand side of Eq. (3), $\left(\Delta \mathrm{H} / \rho \mathrm{c}_{\mathrm{p}}\right)$, is the temperature to which formation of the reaction compound will adiabatically heat up the substrate material: typically, this is for strongly exothermic reactions on the order of $1000 \mathrm{~K}$. Since $\delta=\mathrm{V} \Delta \mathrm{t}$, the second fraction $(\delta / \mathrm{U} \Delta \mathrm{t})$ is the ratio of vertical interfacial reaction product growth rate $(\mathrm{V})$ to the horizontal reaction product growth rate (U), equal to the drop spreading rate. Two estimations can 
be used to show that $\left(T-T_{o}\right)$ is, in practical situations, likely to be sufficiently small not to influence the contact angle.

- First estimation-Suppose that $\mathrm{U}$ and $\mathrm{V}$, reaction product growth velocities along horizontal and vertical directions, are of the same order of magnitude: $\mathrm{U} \Delta \mathrm{t}$ is then roughly equal to $\delta$. Since (i) for dense inorganic substrates, $2 \alpha$ will not fall much below $10^{-6} \mathrm{~m}^{2} \cdot \mathrm{s}^{-1}$ (12), (ii) the reaction layer thickness reached before diffusion slows the rate of reaction significantly, $\delta$, will rarely exceed $1 \mu \mathrm{m}$ $(8,9,13,14)$, and (iii) the triple line velocity $U$ is typically on the order of, or below, $10^{-4} \mathrm{~m} \cdot \mathrm{s}^{-1}$ when wetting is driven by interfacial reaction in sessile drop experiments $(8,9,13,14)$, a is at most on the order of $10^{-3}$. In reactive wetting, therefore, $\mathrm{F}(\mathrm{a})$ will usually not exceed $10^{-3}$, such that $\mathrm{T}$ will not exceed $\mathrm{T}_{\mathrm{o}}$ by more than a few kelvin.

- Second estimation - If we recast Eq. (3) in the form:

$\mathrm{T}-\mathrm{T}_{0}=\beta \delta \mathrm{UH}(\mathrm{a}) \quad$ with $\quad \mathrm{a}=\frac{\mathrm{U}^{2} \Delta \mathrm{t}}{2 \alpha}, \quad \beta=\frac{\Delta \mathrm{H}}{\pi \lambda} \quad$ and $\quad \mathrm{H}(\mathrm{a})=\frac{\mathrm{F}(\mathrm{a})}{\mathrm{a}}=\frac{1}{\mathrm{a}}+\mathrm{e}^{-\mathrm{a}}\left[\mathrm{K}_{0}(\mathrm{a})-\mathrm{K}_{1}(\mathrm{a})\right]$

the relevant function of a, H(a), is now a monotonously decreasing function of a, Fig. (2). Hence, if we can estimate a realistic lower bound for $\mathrm{a}, \mathrm{a}_{\min }, \mathrm{H}\left(\mathrm{a}_{\min }\right)$ provides a realistic upper bound for $\mathrm{H}(\mathrm{a})$. In sessile drop experiments pertinent to wetting of dense substrates by liquid metals, $\alpha$ will not exceed $10^{-4} \mathrm{~m}^{2} \cdot \mathrm{s}^{-1}(12,15)$, and $\mathrm{L}=\mathrm{U} \Delta \mathrm{t}$ will certainly exceed $10^{-9} \mathrm{~m}$. Hence $\mathrm{a}>\mathrm{a}_{\min } \approx 5 \cdot 10^{-6} \mathrm{U}$. Now, at small $\mathrm{a}_{\min }$ (less than about 0.1 , which is certainly the case for realistic values of $\left.\mathrm{U}\right), \mathrm{H}\left(\mathrm{a}_{\min }\right)$ is approximately equal to:

$$
\mathrm{H}\left(\mathrm{a}_{\min }\right)=\frac{1}{\mathrm{a}_{\min }}\left(1-\mathrm{e}^{-\mathrm{a}_{\min }}\right)-\mathrm{e}^{-\mathrm{a}_{\min }}\left[\ln \left(\frac{\mathrm{a}_{\min }}{2}\right)+\gamma\right] \approx 1.11-\ln \left(\mathrm{a}_{\min }\right)
$$

where $\gamma=0.57722$ is Euler's constant. Hence, we can say that $\mathrm{H}(\mathrm{a})$ will, in realistic situations, be less than $\mathrm{H}\left(\mathrm{a}_{\text {min }}\right) \approx 1.11-\ln \left(5 \cdot 10^{-6} \mathrm{U}\right)=13.3-\ln (\mathrm{U})$. For systems considered here (in which substrate materials are based on carbon or oxides and reaction products are composed of refractory ceramics such as $\mathrm{SiC}, \mathrm{Al}_{4} \mathrm{C}_{3}$, or $\mathrm{Cr}_{3} \mathrm{C}_{2}$ ), heats of reactions $\Delta \mathrm{H}$ do not exceed about $5 \cdot 10^{9} \mathrm{~J} \cdot \mathrm{m}^{-3}(16)$. For most inorganic materials, $\pi \lambda \geq 5 \mathrm{~W} \cdot \mathrm{m}^{-1} \cdot \mathrm{K}^{-1}\left(\lambda\right.$ varies for metals between about 25 and $400 \mathrm{~W} \cdot \mathrm{m}^{-1} \cdot \mathrm{K}^{-1}$, and for dense ceramics between about 2 and $\left.50 \mathrm{~W} \cdot \mathrm{m}^{-1} \cdot \mathrm{K}^{-1}(12,15)\right)$. Hence, $\beta$ is at most on the order of $\beta_{\max }=10^{9} \mathrm{~K} \cdot \mathrm{s} \cdot \mathrm{m}^{-2}$. Again, $\delta$, will rarely exceed $\delta_{\max }=10^{-6} \mathrm{~m}$, and in reactive wetting sessile drop experiments $U$ is typically on the order of, or below, $U_{\max }=10^{-4} \mathrm{~m} \cdot \mathrm{s}^{-1}$. Since for $\mathrm{x}<0.368, \mathrm{x} \ln (\mathrm{x})$ is a decreasing function of $\mathrm{x}$, we can conclude that $\mathrm{T}-\mathrm{T}_{\mathrm{o}}<\beta_{\max } \delta_{\max } \mathrm{U}_{\max }\left[13.3-\ln \left(\mathrm{U}_{\max }\right)\right]=2.3$ $\mathrm{K}$, again a few $\mathrm{K}$ in agreement with the first estimation.

It can thus be concluded that conduction through the solid substrate can evacuate heat released by interfacial reactions at, and behind, the triple line, so that the wetting process is essentially isothermal under usual conditions of reactive wetting in sessile drop experiments. We also note that, in this configuration, as $\mathrm{U}$ tends to either zero or infinity with all else constant, the triple line temperature tends towards $\mathrm{T}_{\mathrm{o}}$. At high velocity, the underlying cause is rapid cooling by the incoming solid substrate material, while at low velocity, $\mathrm{T}$ approaches $\mathrm{T}_{\mathrm{o}}$ because for given $\delta, \Delta \mathrm{H}$, and $\Delta \mathrm{t}$, the smaller $\mathrm{U}$ becomes, the smaller the width $(\mathrm{U} \Delta \mathrm{t})$ of the heat-releasing strip of material becomes, and hence the smaller the amount of heat given off at any given instant near the triple line.

\section{$\underline{\text { Wetting in Reactive Infiltration }}$}

This situation, schematically depicted in Fig. 3, is representative of a second important class of dynamic wetting experiments, in which wetting characteristics are derived from measurements of the rate of flow through a thin capillary or, more generally, through a fine porous medium; this situation is found in 


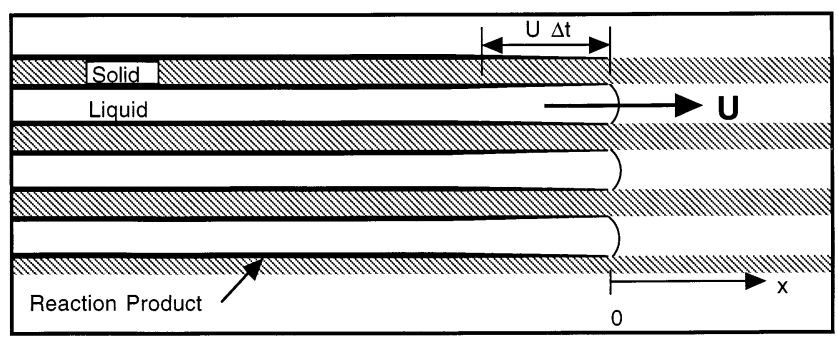

Figure 3. Assumed configuration for reaction layer formation behind the triple line in reactive infiltration.

practice in infiltration processing. We assume that the porous medium is sufficiently fine that it can be assimilated to a continuum, within which a differential volume element can be taken to be sufficiently small on the scale of the macroscopic porous body to be at a homogeneous temperature, yet sufficiently large to comprise a meaningful local average of all phases present. We retain essentially the same assumptions as for the preceding configuration: we consider a fluid flowing at steady state into an initially dry and infinitely long porous medium of the solid substrate material, at a constant velocity $\mathrm{U}$, Fig. 3. We assume that the amount of reaction product is sufficiently small not to represent a significant change in thermal properties of the solid phase present, and that the wetting front of the fluid in the porous medium is well defined, so it can be assimilated to a plane (in other words, we make the slug-flow assumption). In a referential moving at velocity $U$ with the infiltration front, which we take located at $\mathrm{x}=0$, with Ox parallel to $\mathrm{U}$ and with $\mathrm{x}>0$ denoting the uninfiltrated porous medium, heat transport is, in the steady state, constant $\mathrm{U}$, situation considered here, governed by the following equations: (i) for $\mathrm{x}>0$, in the uninfiltrated porous medium,

$$
-\mathrm{V}_{\mathrm{s}} \rho_{\mathrm{s}} \mathrm{c}_{\mathrm{s}} \mathrm{U} \frac{\partial \mathrm{T}}{\partial \mathrm{x}}=\lambda_{\mathrm{s}} \frac{\partial^{2} \mathrm{~T}}{\partial^{2} \mathrm{x}}
$$

where $\mathrm{V}_{\mathrm{s}}$ is the volume fraction solid phase, $\rho_{\mathrm{s}}$ the density of the solid phase, $\mathrm{c}_{\mathrm{s}}$ its heat capacity and $\lambda_{\mathrm{s}}$ the thermal conductivity of the dry porous solid medium; (ii) for $-\mathrm{U} \Delta \mathrm{t}<\mathrm{x}<0$, in the reacting infiltrated porous medium,

$$
-\mathrm{V}_{\mathrm{s}} \rho_{\mathrm{s}} \mathrm{c}_{\mathrm{s}} \mathrm{U} \frac{\partial \mathrm{T}}{\partial \mathrm{x}}=\lambda_{\mathrm{c}} \frac{\partial^{2} \mathrm{~T}}{\partial^{2} \mathrm{x}}+\mathrm{JS}_{\mathrm{v}}
$$

where $\lambda_{\mathrm{c}}$ is the thermal conductivity of the infiltrated composite medium (assumed for simplicity to remain constant), and $S_{\mathrm{V}}$ the liquid/solid interfacial area per unit volume of the composite (which we take constant and equal to the specific surface of the dry porous medium); and (iii) for $\mathrm{x}<-\mathrm{U} \Delta \mathrm{t}$, in the fully reacted infiltrated porous medium,

$$
-\mathrm{V}_{\mathrm{s}} \rho_{\mathrm{s}} \mathrm{c}_{\mathrm{s}} \mathrm{U} \frac{\partial \mathrm{T}}{\partial \mathrm{x}}=\lambda_{\mathrm{c}} \frac{\partial^{2} \mathrm{~T}}{\partial^{2} \mathrm{x}}
$$

Boundary conditions are: (i) for $\mathrm{x}=\infty, \mathrm{T}=\mathrm{T}_{\mathrm{o}}$; and (ii) at $\mathrm{x}=0$ and at $\mathrm{x}=-\mathrm{U} \Delta \mathrm{t}$, that $\mathrm{T}$ and $\lambda_{\mathrm{i}} \partial \mathrm{T} / \partial \mathrm{x}$ be continuous, $\lambda_{\mathrm{i}}$ being the relevant thermal conductivity. Ahead of the infiltration front, the temperature distribution is given by:

$$
\mathrm{T}=\mathrm{K}_{1} \exp \left(\frac{-\mathrm{V}_{\mathrm{s}} \rho_{\mathrm{s}} \mathrm{c}_{\mathrm{s}} \mathrm{Ux}}{\lambda_{\mathrm{s}}}\right)+\mathrm{T}_{0}
$$


while in the reacting region $-\mathrm{U} \Delta \mathrm{t} \leq \mathrm{x} \leq 0$,

$$
\mathrm{T}=\mathrm{K}_{2} \exp \left(\frac{-\mathrm{V}_{\mathrm{s}} \rho_{\mathrm{s}} \mathrm{c}_{\mathrm{s}} \mathrm{Ux}}{\lambda_{\mathrm{c}}}\right)-\frac{\mathrm{JS}_{\mathrm{v}}}{\mathrm{V}_{\mathrm{s}} \rho_{\mathrm{s}} \mathrm{c}_{\mathrm{s}} \mathrm{U}} \mathrm{x}+\mathrm{K}_{3}
$$

and for $\mathrm{x} \leq-\mathrm{U} \Delta \mathrm{t}$,

$$
\mathrm{T}=\mathrm{K}_{4} \exp \left(\frac{-\mathrm{V}_{\mathrm{s}} \rho_{\mathrm{s}} \mathrm{c}_{\mathrm{s}} \mathrm{Ux}}{\lambda_{\mathrm{c}}}\right)+\mathrm{K}_{5}
$$

For this last profile to remain bounded at very long infiltration depths, we take $\mathrm{K}_{4}=0$. Heat flux continuity at $\mathrm{x}=0$, and the condition that at $\mathrm{x}=-\mathrm{U} \Delta \mathrm{t}, \partial \mathrm{T} / \partial \mathrm{x}=0$, then dictate:

$$
\mathrm{K}_{1}=\mathrm{K}_{2}+\frac{\lambda_{\mathrm{c}} \mathrm{JS}_{\mathrm{v}}}{\left(\mathrm{V}_{\mathrm{s}} \rho_{\mathrm{s}} \mathrm{c}_{\mathrm{s}} \mathrm{U}\right)^{2}} \quad \text { and } \quad \mathrm{K}_{2}=-\frac{\lambda_{\mathrm{c}} \mathrm{JS}_{\mathrm{v}}}{\left(\mathrm{V}_{\mathrm{s}} \rho_{\mathrm{s}} \mathrm{c}_{\mathrm{s}} \mathrm{U}\right)^{2}} \exp \left(\frac{-\mathrm{V}_{\mathrm{s}} \rho_{\mathrm{s}} \mathrm{c}_{\mathrm{s}} \mathrm{U}^{2} \Delta \mathrm{t}}{\lambda_{\mathrm{c}}}\right)
$$

so the temperature at $\mathrm{x}=0$ is given by:

$$
\mathrm{T}=\mathrm{T}_{0}+\frac{\mathrm{S}_{\mathrm{v}} \delta \Delta \mathrm{H}}{\mathrm{V}_{\mathrm{s}} \rho_{\mathrm{s}} \mathrm{c}_{\mathrm{s}}}\left\{\frac{1-\mathrm{e}^{-\mathrm{a}^{\prime}}}{\mathrm{a}^{\prime}}\right\} \quad \text { where } \quad \mathrm{a}^{\prime}=\frac{\mathrm{V}_{\mathrm{s}} \rho_{\mathrm{s}} \mathrm{c}_{\mathrm{s}} \mathrm{U}^{2} \Delta \mathrm{t}}{\lambda_{\mathrm{c}}}
$$

The temperature at the triple line depends on a parameter a' similar to parameter a found in the previous case. A plot of the dimensionless function $\mathrm{G}\left(\mathrm{a}^{\prime}\right)=\left[1-\exp \left(-\mathrm{a}^{\prime}\right)\right] / \mathrm{a}^{\prime}$ versus the dimensionless parameter $\mathrm{a}^{\prime}$ is given in Fig. 2. The right-hand side of Eq. (13) goes to zero as $\mathrm{a}^{\prime}$ approaches infinity. Physically, this is because at large $\mathrm{a}^{\prime}$ convection dominates over conduction, such that heat evolved from the interfacial reaction cannot preheat the dry porous solid ahead of the wetting front. Contrary to the previous case, however, $\left(T-T_{o}\right)$ now approaches a finite value as a' falls below 0.1 , this finite value being the temperature to which the interfacial reaction can heat up the solid phase (and the solid phase alone, since in the steady velocity infiltration configuration considered here, the incoming liquid is already heated at the triple line by reaction with solid infiltrated previously) adiabatically to $T=T_{a d}$, given by Eq. (13) with the term between brackets equal to one. The basic underlying reason for this difference in behavior is that, in porous medium infiltration, the quantity of substrate material per unit reaction product volume is limited. In practice, $\mathrm{a}^{\prime}$ can easily fall below 0.1 : with $10^{6} \mathrm{~J} \cdot \mathrm{m}^{-3} \cdot \mathrm{K}^{-1} \leq \rho_{\mathrm{s}}$ $\mathrm{c}_{\mathrm{s}} \leq 5 \times 10^{6} \mathrm{~J} \cdot \mathrm{m}^{-3} \cdot \mathrm{K}^{-1}, \mathrm{~V}_{\mathrm{s}}$ on the order of 0.1 and $\lambda_{\mathrm{C}}>2 \mathrm{~W} \mathrm{~m}^{-1} \mathrm{~K}^{-1}$ (as mentioned above, $\lambda$ varies for metals between about 25 and $400 \mathrm{~W} \cdot \mathrm{m}^{-1} \cdot \mathrm{K}^{-1}$, and for dense ceramics between about 2 and 50 $\mathrm{W} \cdot \mathrm{m}^{-1} \cdot \mathrm{K}^{-1}$ ), it suffices that $\mathrm{U} \leq 10^{-3} \mathrm{~m} \cdot \mathrm{s}^{-1}$ and $\mathrm{U} \Delta \mathrm{t} \leq 10^{-3} \mathrm{~m}$ for $\mathrm{a}^{\prime}$ to be less than 0.1 . The triple line temperature then approximately equals $\mathrm{T}_{\mathrm{ad}}$. If we assume that the reaction product represents one percent of the initial substrate volume, $\mathrm{S}_{\mathrm{v}} \delta$ is on the order of 0.01 . Since values of $\Delta \mathrm{H}\left(\rho_{\mathrm{s}} \mathrm{c}_{\mathrm{s}}\right)^{-1}$ on the order of several thousand kelvin are, as mentioned, not exceptional, with $\mathrm{V}_{\mathrm{s}}$ on the order of $0.1, \mathrm{~T}_{\mathrm{ad}}$ can exceed $\mathrm{T}_{\mathrm{o}}$ by several hundred kelvin. This suffices to affect the local capillary equilibrium significantly, most often towards an improvement of wetting, since a temperature rise in general leads to a decreasing contact angle and an increase in spreading rate. Hence, this simple calculation indicates that in infiltration, heating due to liquid/solid chemical interaction can realistically influence significantly the triple line temperature, even in systems where the amount of solid phase transformed by chemical reaction with the liquid is relatively small.

\section{$\underline{\text { Conclusion }}$}

In the sessile drop configuration and for typical values of spreading rates observed in experiments for reactive metal-ceramic systems we show, using a simple calculation, that heat released by interfacial 
reaction behind the triple line along reacting interfaces is unlikely to cause significant heating of the triple line. Sessile drop experiments thus yield, under usual conditions of reactive wetting, contact angle values which can legitimately be considered isothermal.

Using a similar steady state and steady triple line velocity configuration for infiltration of a porous medium, we show that even limited reaction between solid and liquid can cause sufficient heating at the triple line to affect capillary equilibrium. Transposition of wetting angle values from sessile drop experiments on reactive systems to infiltration must, therefore, be conducted with caution.

\section{Acknowledgments}

A. Mortensen gratefully acknowledges support for this work from the National Science Foundation, grant \# MSS-92-01843, and the French Ministry of Research and Education for a Bourse de Haut Niveau during his sabbatical leave in France.

\section{References}

1. L. A. Aksay, C. E. Hoge, and J. A. Pask, J. Phys. Chem. 78, 1178 (1974).

2. J. V. Naidich, Progress in Surface and Membrane Science, ed. D. A. Cadenhead and J. F. Danielli, pp. 353-484, Academic Press, New York (1981).

3. N. Eustathopoulos, D. Chatain, and L. Coudurier, Mater. Sci. Eng. A135, 83 (1991).

4. N. Eustathopoulos and A. Mortensen, in Fundamentals of Metal Matrix Composites, ed. S. Suresh, A. Mortensen, and A. Needleman, pp. 42-58, Butterworth-Heinemann, Boston (1993).

5. X. B. Zhou and J. T. M. DeHosson, Acta Mater. 44, 421 (1996).

6. B. Drevet, S. Kalegeropoulou, and N. Eustathopoulos, Acta Metall. Mater. 41, 3119 (1993).

7. H. Fujii and H. Nakae, Acta Metall. Mater. 41, 2963 (1993).

8. L. Espié, B. Drevet, and N. Eustathopoulos, Metall. Mater. Trans. 25A, 599 (1994).

9. 9. K. Landry and N. Eustathopoulos, Acta Mater. 44, 3923 (1996).

10. E. B. Dussan V., Annu. Rev. Fluid Mech. 11, 371 (1979)

11. H. S. Carslaw and J. C. Jaeger, Conduction of Heat in Solids, 2nd edn, pp. 266-270, Clarendon Press, Oxford, UK (1959).

12. D. R. Poirier and G. H. Geiger, Transport Phenomena in Materials Processing, pp. 191-209, TMS, Warrendale, PA (1994).

13. B. Drevet, K. Landry, P. Vikner, and N. Eustathopoulos, Scripta Mater. 35, 1265 (1996).

14. K. Landry, C. Rado, R. Voitovitch, and N. Eustathopoulos, Acta Mater. 45, 3079 (1997).

15. Smithells Metals Reference Book, Sections 14 and 27, Butterworth \& Co (Publishers), London (1983).

16. O. Kubaschewski and C. B. Alcock, Metallurgical Thermochemistry, pp. 336-356, Pergamon Press, New York (1979). 